\title{
Single-pixel imaging with deterministic complex-valued sensing matrices
}

\author{
M. Zhao \\ eachzhao@126.com \\ J. Liu \\ S. Chen \\ C. Kang \\ W. $\mathbf{X u}$
}

\author{
College of Information Science Technology, Dalian Maritime University, Dalian 116026, China \\ Department of Mathematics and Systems Science, College of Science, National University of Defense \\ Technology, Changsha 410073, China \\ Research center for space optics engineering, Harbin Institute of Technology, Harbin 150001, China \\ College of Information Science Technology, Dalian Maritime University, Dalian 116026, China \\ College of Information Science Technology, Dalian Maritime University, Dalian 116026, China
}

In this paper, complex deterministic sensing matrices are explored to sample the signals in the single pixel imaging (SPI). A new analysissynthesis scheme is proposed to realize the complex deterministic sensing matrix for the DMD-based SPI. The analysis process divides the complex sensing matrix into real sensing matrix and imaginary sensing matrix, and multiple imaging is performed with these sensing matrices. After synthesizing the real and imaginary measurements, the final image of complex deterministic sensing matrix is reconstructed. The performance of deterministic sensing matrix is investigated through simulation and experiment. Compared with the random sensing matrix, the deterministic sensing matrix gives more favorable reconstructed images.

[DOI: http://dx.doi.org/10.2971/jeos.2015.15041]

Keywords: Single pixel imaging, compressive sensing, deterministic sensing matrix, noiselet

\section{INTRODUCTION}

Single-pixel imaging (SPI) uses detector with one single pixel to acquire object information instead of the millions of pixels used in the imaging sensors of conventional digital cameras. In SPI the two-dimensional spatially coded patterns can be employed before or after the object. Generally, illuminating objects with patterns can be associated with ghost imaging [1, 2] and filtering the light reflected from object with patterns refers to single-pixel camera [3,4].

The signals recorded by the single-pixel detector are correlated with spatially coded patterns so that the objects can be reconstructed from a series of time-synchronized coded samplings. For the intensity-based SPI, the coded patterns are generally a rapidly-shifting sequence of binary (black-and-white) patterns similar to crossword puzzles. This binary pattern can be generated by a spatial light modulator (SLM) or a digital micro-mirror device (DMD).

The emerging theory of compressive sensing (CS) offers a potential solution to SPI, where an image of the scene can be reconstructed from far fewer data/measurements than the number of reconstructed pixels. Several reports have introduced CS into SPI to enhance the performance of SPI. Single-pixel camera is the first application of compressive sensing used in SPI. It utilizes a DMD to displaying a time sequence of random binary sensing patterns, and captures the average light with a single-pixel photodetector. Since then, random sensing matrix has served as the first choice in the imaging process of SPI. With the development of CS theory, deterministic sensing matrices are proposed, and compared with the random sensing matrices, they show more promising performance [5]-[7]: 1) The deterministic matrices are guaranteed to satisfy the coherence condition when they are constructed. 2) Exploiting the structure of deterministic matrices, it is possible to save them in a more storage-saving manner. 3) Its structure may help to devise specific fast recovery algorithm.

In this paper, we present the use of deterministic sensing matrices to sample the signals in the SPI and the aim is to prove the superiority of deterministic sensing matrices in SPI. In addition, the first-hand report, which uses an intensitymodulated DMD to achieve complex-valued sensing matrices, is presented. A combination of DMD-based SPI system and a Noiselet-based complex-valued deterministic sensing matrix is experimented to demonstrate our proposal.

\section{DETERMINISTIC SENSING FOR SPI}

\subsection{Performance of Deterministic Sensing Matrix}

Consider a Length- $N$ signal $x, M$ measurements $y$ are obtained in the data acquisition process. In matrix notation, it 
is described as:

$$
\mathbf{y}=\boldsymbol{\Phi} \mathbf{x}
$$

where $\boldsymbol{\Phi}$ represents an $M \times N$ sensing/measurement matrix. If the signal $x$ is a 2-dimension image, its vector notion $\mathbf{x}$ is obtained by stacking the pixels of the image $x$, and the row of $\boldsymbol{\Phi}$ is the stacking of the corresponding 2-dimension sensing patterns. When $x$ is sparse or can be sparsely represented in some sparsity basis $\Psi$, the CS theory asserts that if the coherence between sensing matrix $\boldsymbol{\Phi}$ and sparsity basis $\Psi$ satisfies certain conditions [8], the signal $x$ can be perfectly reconstructed from y, even if $M \ll N$.

The sensing matrix $\boldsymbol{\Phi}$ plays an important role in CS, and it has a profound impact on the quality of the reconstructed image. To have an insight of the performance of random and deterministic sensing matrices in SPI, we perform a series of simulations. Two sensing matrices are used in the simulation: one of them is the classical Bernoulli random sensing matrix, and the other is deterministic noiselet sensing matrix. Noiselet, which is firstly presented in [9], is perfectly incoherent with the Haar wavelet transform $[10,11]$. This means that we can sample the scene with noiselet sensing matrix, and reconstruct the image via the Haar wavelet basis. The noiselet sensing matrix may be a good candidate for SPI, since: 1 ) the real and imaginary parts of noiselet matrix is binary (only -1 and 1), so it can be efficiently performed by DMD. 2) The noiselet matrix is symmetric and unitary and the generation of noiselet enjoys fast algorithms (computational complexity is $O(N \log N)$, where $N$ is the size of matrix), which can facilitate the process of data processing. 3) For each row of the noiselet matrix, the energy of signal will be spread out, rather than concentrated on several pixels, in the measurement domain, which enables a better recovery condition. 4) The image is always sparse in the wavelet domain, and thus the wavelet is a good sparsity basis.

The original image is a $64 \times 64$ binary image where the letters "DMU" are ones and the background is zero. The measurements $y$ are simulated based on Eq. (1), and added with 1\% Gaussian noise. Figures 1(a) and (b) show the reconstruction results of the two sensing matrices, and Figure 1(c) gives a quantitative comparison of the recovery quality of the two sensing matrices. The reconstruction results of Bernoulli sensing matrix are very blurry, even when subsample rate is close to $1(M=4000)$. On the contrary, the results of noiselet sensing matrix show more excellent performance in the image reconstruction, where $50 \%$ subsample rate $(M=2000)$ can still perfectly reconstruct the Letters. In addition, note that the noiselet sensing matrix shows the advantage of visual quality in its reconstructed image. When the subsample rate is beneath $25 \%$ $(M=1000)$, neither the random matrix nor the noiselet matrix can give a satisfactory result. In Figure 3(c), it is straightforward to find that the deterministic noiselet sensing matrix outperforms the random sensing matrix in terms of PSNR performance.

Although the performance of the deterministic matrices is promising, how to use it in the SPI is a non-trivial work. SPI relies on the DMD to modulate the light. And, DMD can only realize the positive-valued sensing matrix due to its working (a)
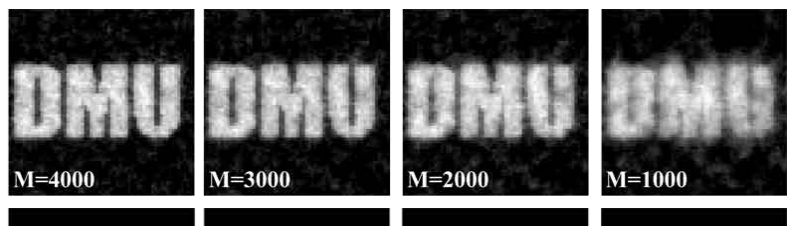

(b)
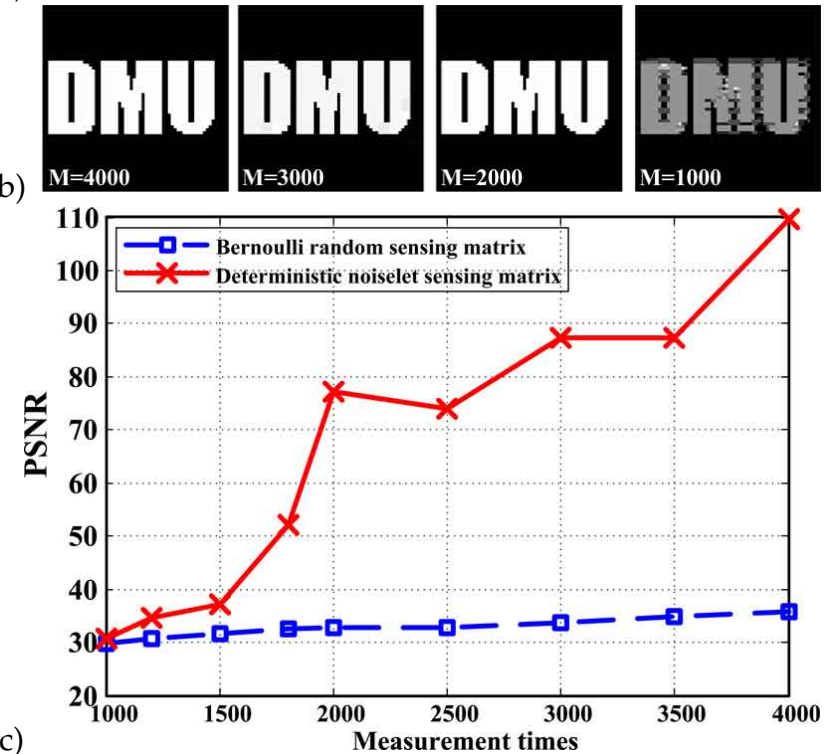

(c)

FIG. 1 Comparison of the recovery quality of random and deterministic sensing matrices in SPI. (a) Bernoulli randomsensing matrix, (b) deterministic noiselet sensing matrix, (c) Peak signal-to-noise ratio (PSNR) performance of the two sensing matrices.

mechanism. But, most of the deterministic matrices are not positive-valued, and some may be complex-valued.

\subsection{The proposed analysis-synthesis scheme}

Since only the positive-valued pattern can be projected by the DMD, a straightforward method is to transform the complex sensing matrix into positive-valued sensing matrix. As we know, the complex number is the sum of a real number and an imaginary number. In the same way, we analyze the complex sensing matrix $\boldsymbol{\Phi}$ into the sum of four matrices, that is:

$$
\boldsymbol{\Phi}=\boldsymbol{\Phi}_{p R}-\boldsymbol{\Phi}_{n R}+\mathbf{i} \boldsymbol{\Phi}_{p I}-\mathbf{i} \boldsymbol{\Phi}_{n I}
$$

where, $\boldsymbol{\Phi}_{p R},-\boldsymbol{\Phi}_{n R}, \mathbf{i} \boldsymbol{\Phi}_{p I}$ and $-\mathbf{i} \boldsymbol{\Phi}_{n I}$ are the positive-real part, negative-real part, positive-imaginary part and negativeimaginary part of complex sensing matrix respectively, and $\boldsymbol{\Phi}_{p R}, \boldsymbol{\Phi}_{n R}, \boldsymbol{\Phi}_{p I}$ and $\boldsymbol{\Phi}_{n I}$ are the positive-valued matrices. When we sample the scene with complex sensing matrix $\boldsymbol{\Phi}$, it can be written as:

$$
\begin{aligned}
\mathbf{y} & =\boldsymbol{\Phi} \mathbf{x} \\
& =\left(\boldsymbol{\Phi}_{p R}-\boldsymbol{\Phi}_{n R}+\mathbf{i} \boldsymbol{\Phi}_{p I}-\mathbf{i} \boldsymbol{\Phi}_{n I}\right) \mathbf{x} \\
& =\boldsymbol{\Phi}_{p R} \mathbf{x}-\boldsymbol{\Phi}_{n R} \mathbf{x}+\mathbf{i} \boldsymbol{\Phi}_{p I} \mathbf{x}-\mathbf{i} \boldsymbol{\Phi}_{n I} \mathbf{x}
\end{aligned}
$$

If we denote $\mathbf{y}_{p R}=\boldsymbol{\Phi}_{p R} \mathbf{x}, \mathbf{y}_{n R}=\boldsymbol{\Phi}_{n R} \mathbf{x}, \mathbf{y}_{p I}=\boldsymbol{\Phi}_{p I} \mathbf{x}$, $\mathbf{y}_{n I}=\boldsymbol{\Phi}_{n I} \mathbf{x}$, where $\boldsymbol{\Phi}_{p R}, \boldsymbol{\Phi}_{n R}, \boldsymbol{\Phi}_{p I}$ and $\boldsymbol{\Phi}_{n I}$ are the positivevalued sensing matrices, then the measurements:

$$
\mathbf{y}=\mathbf{y}_{p R}-\mathbf{y}_{n R}+\mathbf{i y}_{p I}-\mathbf{i} \mathbf{y}_{n I}
$$

This means that we can firstly perform the measurements using the four positive-valued sensing matrices respectively, and 


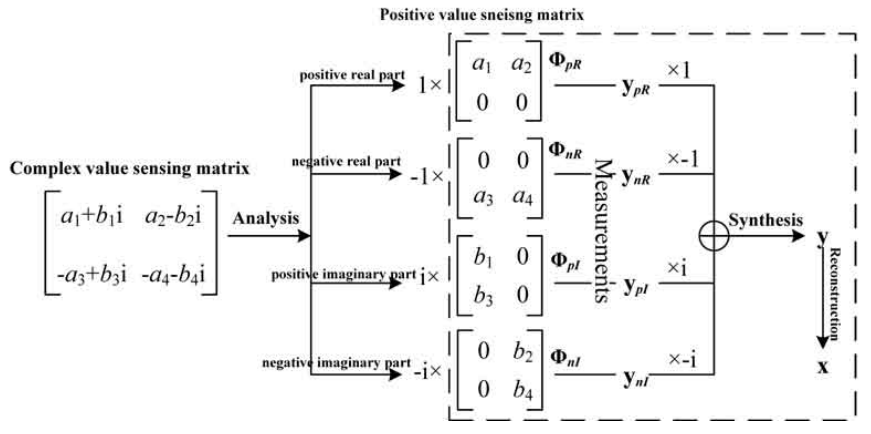

FIC. 2 The proposed analysis-synthesis scheme. Complex sensing matrix is analyzed into four positive-valued sensing matrices, which are used to perform the measurements. Measurements data is synthesized and used to reconstruct the final image.
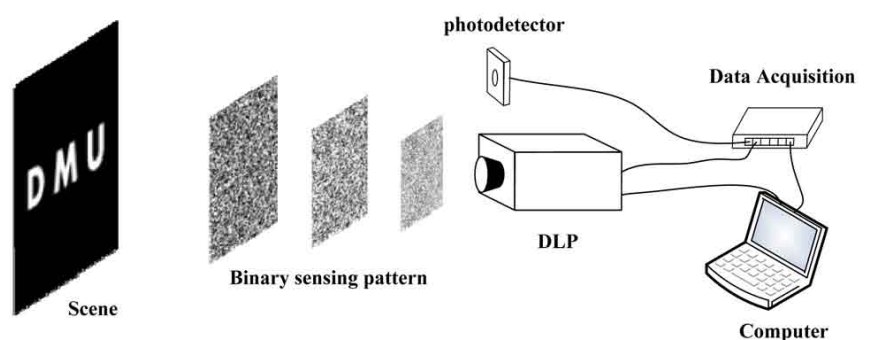

FIG. 3 Experimental setup. A DLP with DMD projects the sensing patterns onto the scene, and the photodetector measures the reflected light synchronously. The computer records the measured data and reconstructs the final image.

then synthesize the collected data by multiplying with $1,-1, i$, and $-i$ and adding up. Since the measurement process of compressive sensing is linear, the synthesized data is same as the acquisition of complex-valued sensing matrix. And thus we can reconstruct the final image with the synthesized data. The proposed AnalysisSynthesis scheme is illustrated in Figure 2.

\section{EXPERIMENTS}

\subsection{Experiments Setup}

The experimental setup is shown in Figure 3. The scene used in the experiment is three printed white letters "DMU" in a black curtain. Digital light projector (DLP) provides spatially incoherent structured illumination on the scene. The DLP we used is the LightCrafter 4500 (produced by Texas Instruments Inc.), whose central core is the digital micro-mirror device (DMD), and it can be controlled to form the desired sensing pattern. For each incident pattern, the total intensity reflected from the scene is synchronously measured by a single-pixel photodetector(produced by Thorlabs Inc., Model:PDA100A). The photodetector signal is converted to digits via a data acquisition device, and recoded by a computer. After all of the sensing patterns are displayed, the measurements are synthesized via Eq. (4) to form the complex data, through which the reconstruction algorithm is performed to obtain the final image.

In the experiment, the nosiselet sensing matrix is employed to demonstrate our proposal. According to the proposed scheme, we can analyze the noiselet sensing matrix into four

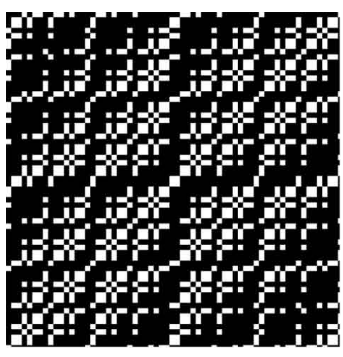

(a)

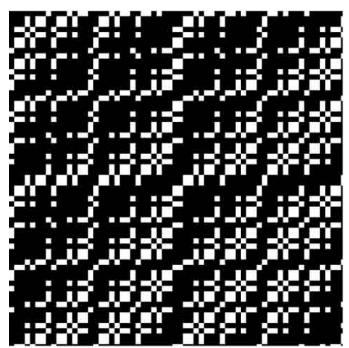

(c)

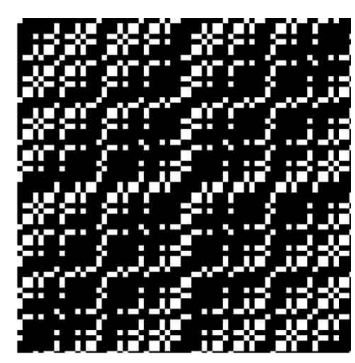

(b)

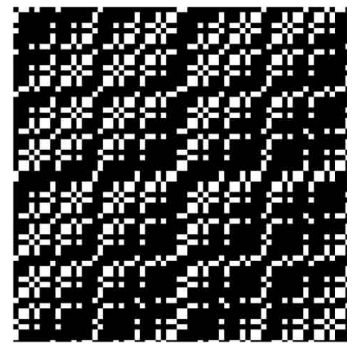

(d)
FIG. 4 A set of positive-valued sensing patterns corresponding to (a) the positive real part, (b) the negative real part, (c) the positive imaginary part, and (d) the negative imaginary part, of the first row of noiselet sensing matrix.

binary valued sensing matrices. Each row of these matrices is the sensing pattern, which is then displayed by DMD. Figure 4 shows an example of the four sensing patterns analyzed from the first row of noiselet sensing matrix.

\subsection{Experimental Results and Discussion}

A total of 3000 noiselet measurement data is collected, where each data is averaged over 5 times measurements to reduce the noise. The image is reconstructed via SpaRSA algorithm [12]. The reconstruction results are shown in Figure 5. As expected, the reconstructed images are sharp. Seen from Figures 5(a) and (b), there is little visual difference between 3000 measurements and 2000 measurements. It means that 2000 measurements are sufficient to recover the "letter" image, and there are at least 1000 redundant measurements in the total data. Such conclusion is matched with our simulation results. Besides, it is obvious that the image quality is decayed with the decreasing number of noiselet measurement data. We can see from Figures 5(c) and (d) that the image artifacts become increasingly apparent when the measurements $M$ is lower than 1500. In order to achieve a faithful reconstruction, the measurements M shouldn't be less than 1500 .

For comparison, the Bernoulli random sensing matrix is performed in the experiment. The reconstructed results are shown in Figure 6. Similar to the simulations, the reconstructed images are still blurry. In addition, the images become more blurred with respect to the decrease of the measurements M. Comparing Figure 5 with Figure 6, we can find that the noiselet sensing matrix is able to recover the sharp features of scene, and thus presents more satisfactory results than the random sensing matrix. In conclusion, deterministic sensing matrix has shown excellent performance in experiment. We expect that more deterministic sensing matrices will be used in SPI. 

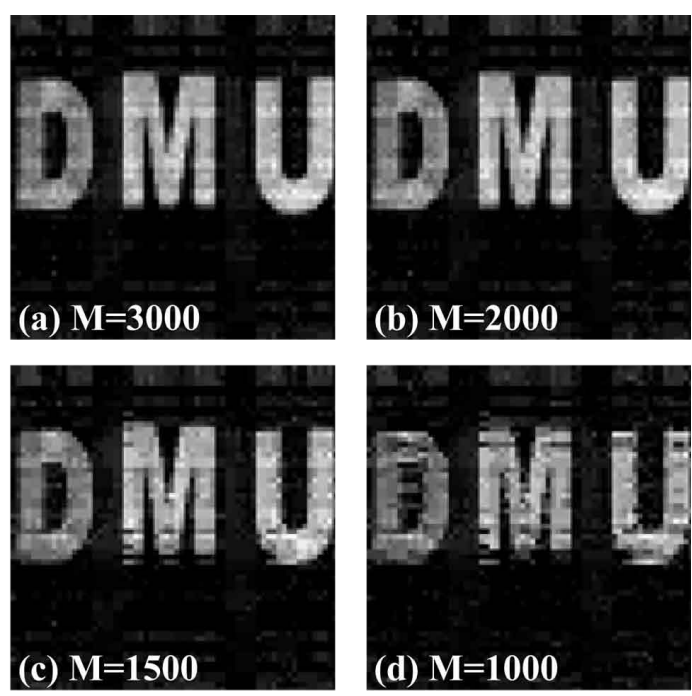

FIG. 5 Reconstructed imagesof $M$ measurement data with noiselet sensing matrix.
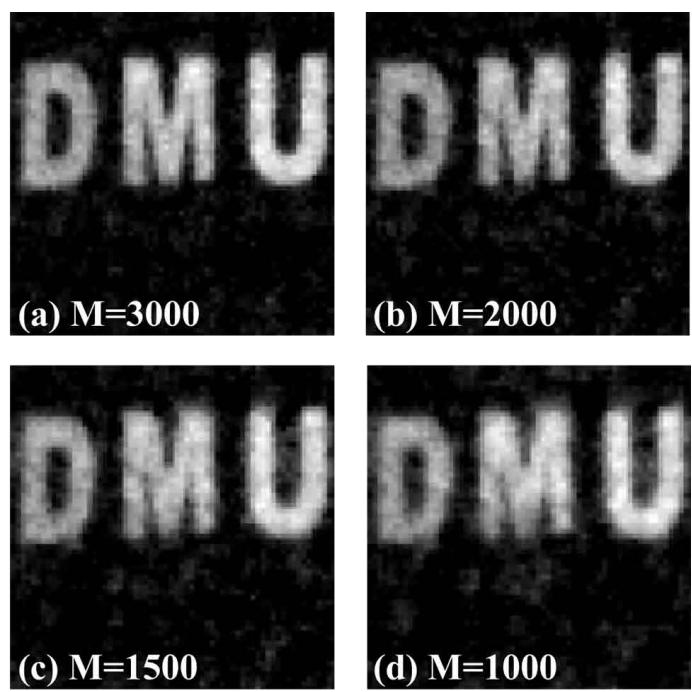

FIG. 6 Reconstructed images of $M$ measurement data with random sensing matrix.

We can see from Figure 1, Figure 5 and Figure 6 that the experimental results are degraded comparing to the simulated results, whether it is the random sensing matrix or deterministic sensing matrix. This is because the light projector is not perfect: firstly, it can't project the absolute blackness; secondly, its light may flicker. All these errors are incorporated into the final image reconstruction, and result in the degradation.

Finally, we should note that the proposed analysis - synthesis scheme requires fourfold measurements compared to the classical DMD-based single pixel imaging, since we have divided the sensing matrix into 4 sensing matrices. However, our scheme can be paralleled. For example, we can use four pairs of DMD and photodetector to build up four single-pixel cameras, where each DMD realizes one part of the noiselet sensing patterns. And then the collected data of the four single-pixel cameras is synthesized, and used to reconstruct the final image.

\section{CONCLUSION}

In this paper, the deterministic sensing matrices are explored to sample the signals in the single pixel imaging. A new analysis-synthesis scheme is proposed to realize the complex deterministic sensing matrix for the DMD-based SPI. And the experiments based on our scheme are setup to investigate the performance of the Noiselet-based deterministic sensing matrix. The simulation and experiments demonstrate that the deterministic sensing matrix outperforms the random sensing matrix.

\section{ACKNOWLEDGEMENT}

This work is supported by the Fundamental Research Fund for the Central Universities of China (No.01750303), the National Science \& Technology Pillar Program (No. 2014BAB12B03 and No. 2014BAB12B04), China National Natural Science Foundation (No. 61201332).

\section{References}

[1] B. Sun, S. S. Welsh, M. P. Edgar, J. H. Shapiro, and M. J. Padgett, “Normalized ghost imaging," Opt. Express 20(15), 16892-16901 (2012).

[2] B. Sun, M. P. Edgar, R. Bowman, L. E. Vittert, S. Welsh, A. Bowman, and M. J. Padgett, " $3 \mathrm{D}$ Computational Imaging with Single-Pixel Detectors," Science 340, 844-847 (2013).

[3] M. F. Duarte, M. A. Dav M. F. Duarte, M. A. Davenport, D. Takhar, J. N. Laska, T. Sun, et al., "Single-pixel imaging via compressive sampling," IEEE Signal Proc. Mag. 25(2), 83-91 (2008).

[4] D. Takhar, J.N. Laska, M. B. Wakin, M. F. Duarte, D. Baron, S. Sarvotham, K. F. Kelly, R. G. Baraniuk, "A New Compressive Imaging Camera Architecture using 0ptical-Domain Compression," Proc. SPIE 6065, 606509 (2006).

[5] M. M. Mohades, A. Mohades, and A. Tadaion, "A Reed-Solomon Code Based Measurement Matrix with Small Coherence," IEEE Signal Proc. Let. 21(7), 839-843 (2014).

[6] S. Li, and G. Ge, "Deterministic Sensing Matrices Arising from Near Orthogonal Systems," IEEE T. Inform. Theory 60(4), 2291-2302 (2014).

[7] R. Calderbank, S. Howard, and S. Jafarpour, "Construction of a large class of deterministic sensing matrices that satisfy a statistical isometry property," IEEE J. Sel. Top. Signa. 4(2), 358-374 (2010).

[8] Y. C. Elar, and G. Kutyniok, Compressed Sensing Theory and Applications (Cambridge Press, Cambridge, 2012).

[9] R. Coifman, F. Geshwind, and Y. Meyer, "Noiselets," Appl. Comput. Harmon. A. 10, 27-44 (2001).

[10] T. Tuma, and P. Hurley, "On the incoherence of noiselet and Haar bases," http://dsp.rice.edu/sites/dsp.rice.edu/files/cs/ noiselets.pdf).

[11] E. Candes, and J. Romberg, "Sparsity and Incoherence in Compressive Sampling," Inverse Probl. 23, 969-985 (2007).

[12] S. J. Wright, R. D. Nowak, and M. A. T. Figueiredo, "Sparse Reconstruction by Separable Approximation," IEEE T. Signal Proces. 57(7), 2479-2493 (2009). 